TRANSACTIONS OF THE

AMERICAN MATHEMATICAL SOCIETY

Volume 349, Number 5, May 1997, Pages 2035-2048

S $0002-9947(97) 01714-5$

\title{
PERIODIC SOLUTIONS TO NONLINEAR ONE DIMENSIONAL WAVE EQUATION WITH $X$-DEPENDENT COEFFICIENTS
}

\author{
V. BARBU AND N. H. PAVEL
}

\begin{abstract}
This paper deals with $t$-periodicity and regularity of solutions to
the one dimensional nonlinear wave equation with $x$-dependent coefficients
\end{abstract}

\section{INTRODUCTION}

This paper deals with the study of $T$-periodic solutions for the nonlinear one dimensional wave equation with $x$-dependent coefficients:

$$
\begin{aligned}
& u(x) y_{t t}-\left(u(x) y_{x}\right)_{x}+g(y)=f(x, t), \quad 0<x<\pi, \quad t \in \mathbb{R}, \\
& y(0, t)=y(\pi, t)=0, \\
& y(x, t+T)=y(x, t), \quad 0<x<\pi, \quad t \in \mathbb{R},
\end{aligned}
$$

under the following hypotheses:

(H1) $u \in H^{2}(0, \pi) ; u(x) \geq 1, \forall x \in[0, \pi]$ and

$$
\rho=\operatorname{ess} \inf \eta_{u}(x)>0,
$$

where

$$
\eta_{u}(x)=\frac{1}{2} \frac{u^{\prime \prime}}{u}-\frac{1}{4}\left(\frac{u^{\prime}}{u}\right)^{2} .
$$

(H2) The function $g: \mathbb{R} \rightarrow \mathbb{R}$ is continuous and nondecreasing, and

$$
|g(y)-g(z)| \leq \gamma|y-z|, \quad y, z \in \mathbb{R},
$$

for some $\gamma>0$.

Another assumption relating $f$ and $g$ is given in Section 3 (Hypothesis (H3)).

Equation (1.1) is a nonlinear model for the forced vibrations of a nonhomogeneous string as well as for propagation of waves in nonisotropic media (see e.g. [2]). More precisely, the displacement $y(z, t)$ at depth $z$ and time $t$ in the case of plane seismic waves is described by the equation

$$
\rho(z) y_{t t}-\left(\mu(z) y_{z}\right)_{z}=0
$$

with some boundary conditions in $z$ and initial conditions in $t$, where $\rho(z)$ is the rock density at depth $z$ and $\mu(z)$ is the elasticity coefficient at $z$.

Received by the editors April 18, 1995 and, in revised form, December 4, 1995.

1991 Mathematics Subject Classification. Primary 35L70, 35B10, 35L05.

Key words and phrases. Forced vibrations of nonhomogeneous strings, propagation of seismic waves, eigenvalues and eigenfunctions, Fourier series, subdifferentials, maximal monotone operators, Sobolev spaces.

This research was carried out while the first author was visiting Ohio University.

(C) 1997 American Mathematical Society 
By a change of variable $z \rightarrow x$ given by $x=\int_{0}^{z}\left(\frac{\rho(s)}{\mu(s)}\right)^{1 / 2} d s$ the equation (1.5) leads to (1.1) (with $f=g=0$ and $u=(\rho \mu)^{1 / 2}$ - the acoustic impedance function).

An inverse problem for (1.1) was recently studied by the authors in [3] for $g=0$ and $T=(2 k+1) / 2 p ; k=0,1, \ldots ; p=1,2, \ldots$ The special case $u \equiv$ constant was extensively studied in the last years beginning with the classical paper of $P$. Rabinowitz [9] and more recently by Brézis and Nirenberg [5], Bahri and Brézis [1]. For a complete reference we refer to the survey of Brézis [4]. In these papers $T$ is a rational multiple of $\pi$. The case in which $T$ can be an irrational multiple of $\pi$ (i.e., $T=\pi \beta$ for some irrational number $\beta$ ) has been investigated by McKenna [8]. (See also [7].) To our best knowledge there are no previous results on the more general case of (1.1) considered here. The main result of this paper is Theorem 3.1, which is an extension of results obtained by Bahri and Brézis [1].

The main notations in this paper are given below:

$Q=(0, \pi) \times(0, T)$

The inner product in $L^{2}(Q)$ is denoted by $\langle\cdot, \cdot\rangle$ and is defined as

$$
\langle f, g\rangle=\int_{Q} u(x) f(x, t) \bar{g}(x, t) d x d t, \quad f, g \in L^{2}(Q) .
$$

Accordingly,

$$
|f|_{L^{2}(Q)}^{2}=|f|^{2}=\int_{Q} u(x)|f(x, t)|^{2} d x d t .
$$

In addition:

$$
|f|_{L^{\infty}(Q)}=|f|_{\infty}=\operatorname{ess} \sup \{|f(x, t)| ;(x, t) \in Q\}, \quad f \in L^{\infty}(Q) .
$$

$H^{1}(Q)$ is the usual Sobolev space, $\mathbb{Z}$ is the set of all integers and $\mathbb{N}$ is the set of all positive integers. Throughout this paper $T$ is a rational multiple of $\pi$. We will write this as

$$
T=2 \pi \frac{p}{q}, \quad \text { with } p, q \text { relatively prime positive integers. }
$$

If $S$ is a closed subset of $L^{2}(Q)$, then $S^{\perp}$ stands for the orthogonal complement of $S$ in $L^{2}(Q)$. Note that in (H1) we can replace $u(x) \geq 1$ by $u(x) \geq a>0$. In this case $\gamma$ in (3.4) must be replaced by $a^{-1} \gamma$ and $\gamma<\alpha$ in Theorem 3.1 by $a^{-1} \gamma<\alpha$.

\section{The linear operator associated With EQUation (1.1)}

We shall use the complete orthonormal system of eigenfunctions $\left\{\psi_{m} \varphi_{n} ; m \in\right.$ $\mathbb{Z}, n \in \mathbb{N}\}$ as a basis for $L^{2}(Q)$, where $[10$, p. 88]

$$
\psi_{m}(t)=T^{-\frac{1}{2}} e^{i \mu_{m} t}, \quad \mu_{m}=2 m \pi T^{-1}, \quad m \in \mathbb{Z},
$$

and $\lambda_{n}, \varphi_{n}$ are given by the Sturm-Liouville problem

$$
-\left(u \varphi_{n}^{\prime}\right)_{x}=u \lambda_{n}^{2} \varphi_{n}, \quad \varphi_{n}(0)=\varphi_{n}(\pi)=0, \quad n \in \mathbb{N},
$$

with $\varphi^{\prime}=\varphi_{x}=\frac{d \varphi}{d x}$.

It is known that $\lambda_{n}$ is (increasingly) convergent to $+\infty$ as $n \rightarrow+\infty$. The inner product in $L^{2}(0, \pi)$ is defined by

$$
\langle\eta, \theta\rangle=\int_{0}^{\pi} u(x) \eta(x) \bar{\theta}(x) d x, \quad \eta, \theta \in L^{2}(0, \pi) .
$$

Accordingly, $\left|\varphi_{n}\right|_{L^{2}(0, \pi)}^{2}=\int_{0}^{\pi} u(x) \varphi_{n}^{2}(x) d x=1$. 
Lemma 2.1. Let u satisfy (H1). Then the eigenvalues $\lambda_{n}^{2}$ of problem (2.2) have the form

$$
\begin{aligned}
& \lambda_{n}=n+\theta_{n} \quad \text { with } \theta_{n} \rightarrow 0 \text { as } n \rightarrow+\infty \\
& 0<\frac{b}{n} \leq \sqrt{n^{2}+\rho}-n \leq \theta_{n} \leq \sqrt{n^{2}+\rho_{1}}-n \leq \sqrt{1+\rho_{1}}-1,
\end{aligned}
$$

where

$$
b=\sqrt{1+\rho}-1, \quad \rho_{1}=\frac{2}{\pi} \int_{0}^{\pi} \eta_{u}(x) d x,
$$

with $\eta_{u}$ as defined in (1.3)

The proof of this lemma is based on the following fact.

Lemma 2.2. Let $\eta$ be a real function in $L^{2}(0, \pi)$ and let $\lambda_{1}^{2}<\lambda_{2}^{2}<\cdots$ and $z_{1}, z_{2}, \ldots$ denote the eigenvalues and real eigenfunctions of the Sturm-Liouville problem

$$
z_{n}^{\prime \prime}(x)+\left(\lambda_{n}^{2}-\eta(x)\right) z_{n}(x)=0, \quad z_{n}(0)=z_{n}(\pi)=0 .
$$

Then the following inequalities hold:

$$
n^{2}+\rho \leq \lambda_{n}^{2} \leq \lambda+n^{2}+\frac{2}{\pi} \int_{0}^{\pi}(\lambda-\eta(x)) d x
$$

for all $\lambda<\lambda_{n}^{2}, n=1,2, \ldots$, where $\rho$ is given in (1.2) and

$$
(h(x))_{+}=\max (h(x), 0) ; \quad(h(x))_{-}=(h(x))_{+}-h(x) .
$$

Proof. By the substitution $z_{n}(x)=(u(x))^{1 / 2} \varphi_{n}(x)$, problem (2.2) is equivalent to (2.6) with $\eta=\eta_{u}(x)$ given by (1.3). In view of (1.2) one can prove that $\lambda_{n}^{2}>\rho$ for all $n=1,2, \ldots$ (Indeed, assume for a contradiction that $\lambda_{1}^{2} \leq \rho$. Then $\lambda_{1}^{2} \leq \eta(x)$ for a.e. $x \in(0, \pi)$. Multiplying $(2.6)$ by $z_{1}$ and integrating over $(0, \pi)$, one concludes that $z_{1} \equiv 0$, which is absurd.) Therefore, we have $0<\rho<\lambda_{1}^{2}<$ $\lambda_{2}^{2}<\cdots<\lambda_{n}^{2}<\cdots$. The lower bound for $\lambda_{n}^{2}$ in (2.8), i.e., $n^{2}+\rho \leq \lambda_{n}^{2}$ for all $n \in \mathbb{N}$, is easy to prove. Indeed, $z_{n}$ has exactly $n-1$ zeros in $(0, \pi)$, denoted say by $0<a_{1}<a_{2}<\cdots<a_{n-1}<\pi, 0=a_{0}, \pi=a_{n}$. In view of (2.6) we have

$$
\begin{aligned}
& \left(\lambda_{n}^{2}-\rho\right) \int_{a_{i-1}}^{a_{i}} z_{n}^{2}(x) d x=\int_{a_{i-1}}^{a_{i}}\left(z_{n}^{\prime}(x)\right)^{2}+(\eta(x)-\rho) z_{n}^{2}(x) d x, \\
& \int_{a_{i-1}}^{a_{i}} z_{n}^{2}(x) d x \leq \pi^{-2}\left(a_{i}-a_{i-1}\right)^{2} \int_{a_{i-1}}^{a_{i}}\left(z_{n}^{\prime}(x)\right)^{2} d x, \quad i=1, \ldots, n,
\end{aligned}
$$

which yields

$$
\int_{a_{i-1}}^{a_{i}}\left(z_{n}^{\prime}(x)\right)^{2} d x \leq\left(\lambda_{n}^{2}-\rho\right) \pi^{-2}\left(a_{i}-a_{i-1}\right)^{2} \int_{a_{i-1}}^{a_{i}}\left(z_{n}^{\prime}(x)\right)^{2} d x ;
$$

so, dividing by $\int_{a_{i-1}}^{a_{i}}\left(z_{n}^{\prime}\right)^{2} d x\left(a_{i}-a_{i-1}\right)^{2}$ and summing over $i$, we get

$$
\sum_{i=1}^{n} \frac{1}{\left(a_{i}-a_{i-1}\right)^{2}} \leq \pi^{-2}\left(\lambda_{n}^{2}-\rho\right) n
$$

But $\min \left\{\sum_{i=1}^{n} \frac{1}{x_{i}^{2}} ; x_{i}>0, x_{1}+\cdots+x_{n}=\pi\right\}=n\left(\frac{n}{\pi}\right)^{2}$ and it is assumed for $x_{1}=x_{2}=\cdots=x_{n}=\frac{\pi}{n}$, so (2.9) implies $n^{2} \leq \lambda_{n}^{2}-\rho$. The upper bound for $\lambda_{n}^{2}$ in (2.7) is taken from Theorem 3.2 in [6] (with $a=0, b=\pi, q(x)=\eta(x)$, and $\lambda_{n+1}^{2}$ in place of $\lambda_{n}$ ). 
Proof of Lemma 2.1. For $\lambda=0$, (2.7) becomes $n^{2}+\rho \leq \lambda_{n}^{2} \leq n^{2}+\rho_{1}$, which leads to $\lambda_{n}=n+\theta_{n}$ with $\sqrt{n^{2}+\rho}-n \leq \theta_{n} \leq \sqrt{n^{2}+\rho_{1}}-n$. This proves that $\theta_{n}>0$, $\theta_{n} \rightarrow 0$ as $n \rightarrow+\infty$, and (2.4) holds. The proof is complete.

Definition 2.1. A function $y \in L^{2}(Q)$ is said to be a weak solution of the problem

$$
\begin{aligned}
& u y_{t t}-\left(u y_{x}\right)_{x}=f(x, t) \quad \text { in } Q, \quad f \in L^{2}(Q), \\
& y(0, t)=y(\pi, t)=0, \quad t \in[0, T], \\
& y(x, 0)=y(x, T), \quad y_{t}(x, 0)=y_{t}(x, T), \quad x \in[0, \pi],
\end{aligned}
$$

if

$$
\int_{Q} y\left(u \varphi_{t t}-\left(u \varphi_{x}\right)_{x}\right) d x d t=\int_{Q} f \varphi d x
$$

for all $\varphi \in C_{\pi}^{2}(\bar{Q})$, where

$C_{\pi}^{2}(\bar{Q})=\left\{\varphi \in C^{2}(\bar{Q}), \varphi(0, t)=\varphi(\pi, t)=0, \varphi(x, 0)=\varphi(x, T), \varphi_{t}(x, 0)=\varphi_{t}(x, T)\right\}$.

Conversely, a weak solution of class $H^{2}(Q)$ satisfies (2.10) in classical sense.

Set

$$
D(\tilde{A})=\left\{y \in L^{2}(Q) ; \text { there is } f \in L^{2}(Q) \text { such that (2.11) holds }\right\} .
$$

Define $\tilde{A}: D(\tilde{A}) \rightarrow L^{2}(Q)$ and $A$ by

$$
\tilde{A} y=f, \quad y \in \tilde{D(A)} ; \quad A=u^{-1} \tilde{A} .
$$

Clearly, $D(A)=D(\tilde{A})$ contains the null function of $L^{2}(Q)$, and for each $y \in D(A)$ there is precisely one $f \in L^{2}(Q)$ satisfying (2.11) (due to the density of $C_{\pi}^{2}(\bar{Q})$ in $\left.L^{2}(Q)\right)$. Therefore the operator $A$ defined by $(2.13)-(2.14)$ is a linear operator from $L^{2}(Q) \rightarrow L^{2}(Q)$, and $(2.11)$ can be rewritten as

$$
\int_{Q} y A_{0} \varphi d x d t=\int_{Q} \tilde{A} y \varphi d x d t=\int_{Q} u A y \varphi d x d t, \quad \varphi \in C_{\pi}^{2}(\bar{Q}), y \in D(A),
$$

where

$$
A_{0} \varphi=u \varphi_{t t}-\left(u \varphi_{x}\right)_{x}, \quad \varphi \in C_{\pi}^{2}(\bar{Q}) .
$$

The operator $\tilde{A}$ defined by (2.13) and (2.14) is said to be the linear operator associated with (1.1). In what follows the main properties of $A$ are given (with $T$ as in (1.10)).

Lemma 2.3. Let u satisfy (H1). Then the null space $N(A)$ of $A$ is finite dimensional and it is given by

$$
N(A)=\operatorname{span}\left\{\psi_{m} \varphi_{n} ; m \in \mathbb{Z}, n \in \mathbb{N} \text { with } \lambda_{n}=\left|\mu_{m}\right|\right\}=N(\tilde{A}) .
$$

If $\rho_{1}<2 p^{-1}+p^{-2}$, then $N(A)$ is zero (where $\rho_{1}$ is given in (2.5)).

Proof. Let $y \in N(A)$, i.e., $A y=0$, and let $y_{m n}$ be the Fourier coefficients of $y$ in $L^{2}(Q)$, i.e.,

$$
\begin{aligned}
& y=\sum_{m, n} y_{m n} \psi_{m}(t) \varphi_{n}(x), \quad y_{m n}=\int_{Q} u(x) y(x, t) \varphi_{n}(x) \bar{\psi}_{m}(t) d x d t \\
& \int_{Q} y A_{0}(\varphi) d x=0, \quad \forall \varphi \in C_{\pi}^{2}(\bar{Q}) .
\end{aligned}
$$


Substituting $\varphi=\varphi_{n} \bar{\psi}_{m}$ into (2.18), we see that

$$
y \in N(A) \text { if and only if }\left(\lambda_{n}^{2}-\mu_{m}^{2}\right) y_{m n}=0,
$$

which implies (2.16). Moreover it is easy to check that the equality

$$
\lambda_{n}=\left|\mu_{m}\right| \text {, i.e., } n+\theta_{n}=\frac{2|m| \pi}{T}, \quad n \in \mathbb{N}, m \in \mathbb{Z},
$$

with $T=2 \pi \frac{p}{q}$ can be valid for at most a finite numbers of pairs $(m, n)$ Indeed, (2.20) means

$$
p n+p \theta_{n}=|m| q
$$

with $0<p \theta_{n}<1$ for $n$ sufficiently large, so (2.21) has at most a finite number of solutions $(m, n)$. If $\rho_{1}<2 p^{-1}+p^{-2}$, then on the basis of $(2.4)$ it follows that

$$
0<p \theta_{n} \leq p\left(\sqrt{1+\rho_{1}}-1\right)<1 \text { for all } n \in \mathbb{N},
$$

so $(2.21)$ has no solutions $(m, n)$. This completes the proof. Note that

$$
N(A)^{\perp}=\operatorname{span}\left\{\psi_{m} \varphi_{n} ; m \in \mathbb{Z}, n \in \mathbb{N} \text { with } \lambda_{n} \neq\left|\mu_{m}\right|\right\} .
$$

The main result of this section is

Proposition 2.1. Let $T$ be a rational multiple of $\pi$ and let $u$ satisfy $(\mathrm{H} 1)$. Then $R(A)$ is closed in $L^{2}(Q), A$ is self-adjoint and $A^{-1} \in L(R(A), R(A))$. Moreover, we have

$$
\left|A^{-1} f\right| \leq d^{-1}|f|, \quad \forall f \in R(A),
$$

where $d=\inf \left\{\left|\lambda_{n}^{2}-\mu_{m}^{2}\right|, \lambda_{n} \neq\left|\mu_{m}\right|\right\}$,

$$
\begin{aligned}
& \left\langle A^{-1} f, f\right\rangle \geq-\alpha^{-1}|f|^{2}, \quad \forall f \in R(A), \\
& \langle A y, y\rangle \geq-\alpha^{-1}|A y|, \quad y \in D(A),
\end{aligned}
$$

where $\alpha=\inf \left\{\mu_{m}^{2}-\lambda_{n}^{2} ;\left|\mu_{m}\right|>\lambda_{n}\right\}$,

$$
\begin{aligned}
& \left|A^{-1} f\right|_{L^{\infty}(Q)} \leq C|f|, \quad \forall f \in R(A), \\
& \left|A^{-1} f\right|_{H^{1}(Q)} \leq C|f|_{H^{1}(Q)}, \quad \forall f \in H^{1}(Q) \cap R(A), \\
& R(A)=N(A)^{\perp} ; \quad L^{2}(Q)=N(A) \oplus R(A)=N(\tilde{A}) \oplus u^{-1} R(\tilde{A}) .
\end{aligned}
$$

Proof. Let $y_{m n}$ and $f_{m n}$ be the Fourier coefficients of $y$ and $f$ respectively, with respect to the orthonormal system $\left\{\psi_{m} \varphi_{m}\right\}$ defined by (2.1) and (2.2), i.e.,

$$
y=\sum_{m, n} y_{m n} \psi_{m} \varphi_{n} ; \quad f=\sum_{m, n} f_{m n} \psi_{m} \varphi_{n} ; \quad \sum_{m, n} f_{m n}^{2}=\int_{Q} u f^{2}=|f|^{2} .
$$

It follows that $A y=f$ (i.e., (2.11) holds) if and only if

$$
\left(\lambda_{n}^{2}-\left|\mu_{m}\right|^{2}\right) y_{m n}=f_{m n}, \quad m \in \mathbb{Z}, n \in \mathbb{N} .
$$

This shows that a necessary condition for the equation $A y=f$ to have a solution $y$ is $f \in N(A)^{\perp}$, i.e., $f_{m n}=0$ for all $(m, n)$ such that $\lambda_{n}=\left|\mu_{m}\right|$. We now prove that this condition is also sufficient. In other words we will prove that the series

$$
\sum_{\lambda_{n} \neq\left|\mu_{m}\right|}\left|y_{m n}\right|^{2} \text { with } y_{m n}=\frac{f_{m n}}{\lambda_{n}^{2}-\mu_{m}^{2}}, \quad \lambda_{n} \neq\left|\mu_{m}\right|,
$$


is convergent (i.e., $R(A)=N(A)^{\perp}$ and (2.27) holds). The key fact is

$$
\inf _{\lambda_{n} \neq\left|\mu_{m}\right|}\left\{\left.\left|\lambda_{n}^{2}-\right| \mu_{m}\right|^{2} \mid\right\}=d>0 .
$$

Indeed, $\lambda_{n}=n+\theta_{n}$ with $\theta_{n} \rightarrow 0$ as $n \rightarrow \infty, \theta_{n} \geq \frac{b}{n}>0$ (as in Lemma 2.1) and $\mu_{m}=|m| \frac{q}{p}$, so

$$
\left.\left|\lambda_{n}^{2}-\right| \mu_{m}\right|^{2}\left|=p^{-2}\right| p n-|m| q+p \theta_{n} \mid\left(p n+|m| q+\rho \theta_{n}\right)
$$

with

$$
\left(p n+|m| q+p \theta_{n}\right) \geq \delta(n+|m|), \quad \delta=\min \{p, q\} .
$$

If $p n=|m| q$, then

$$
\left.\left|\lambda_{n}^{2}-\right| \mu_{m}\right|^{2} \mid \geq n \theta_{n} \geq b
$$

If $p n \neq|m| q$, we derive

$$
\inf _{\substack{\lambda_{n} \neq\left|\mu_{m}\right| \\ p n \neq|m| q}}|p n-| m\left|q+p \theta_{n}\right| \geq c>0
$$

(for some $c>0$ ) due to $\lim _{n \rightarrow \infty} \theta_{n}=0$.

Inequalities (2.34) and (2.35) imply (2.31). We now have on the basis of (2.30) and $(2.31)$

$$
\sum_{\lambda_{n} \neq\left|\mu_{m}\right|}\left|y_{m n}\right|^{2} \leq \frac{1}{d^{2}} \sum_{m, n}\left|f_{m n}\right|^{2}=\frac{1}{d^{2}}|f|^{2},
$$

so (2.23) holds.

Inequality (2.24) is immediate. Indeed, by (2.29)

$$
\left\langle A^{-1} f, f\right\rangle_{L^{2}(Q)}=\sum_{\lambda_{n} \neq\left|\mu_{m}\right|} \frac{f_{m n}^{2}}{\lambda_{n}^{2}-\left|\mu_{m}\right|^{2}} \geq \sum_{\lambda_{n}<\left|\mu_{m}\right|} \frac{f_{m n}^{2}}{\lambda_{n}^{2}-\left|\mu_{m}\right|^{2}},
$$

which yields (2.24). In addition, (2.29) implies that $A$ is symmetric.

If $f \in H^{1}(Q) \cap R(A)$, then the distributional derivative $y_{x}$ of $y=A^{-1} f$ is given by

$$
y_{x}=\sum_{\lambda_{n} \neq\left|\mu_{m}\right|} y_{m n} \psi_{m} \varphi_{n}^{\prime},
$$

where $\left\{\varphi_{n}^{\prime}\right\}$ is orthogonal in $L^{2}(0, \pi)$ and

$$
\left|\varphi_{n}^{\prime}\right|_{L^{2}(0, \pi)}^{2}=\int_{0}^{1} u(x)\left(\varphi_{n}^{\prime}\right)^{2} d x=-\int_{0}^{1} \varphi_{n}\left(u \varphi_{n}^{\prime}\right)_{x} d x=\lambda_{n}^{2} .
$$

Therefore

$$
\begin{aligned}
& \left|y_{x}\right|_{L^{2}(Q)}^{2}=\sum_{\lambda_{n} \neq\left|\mu_{m}\right|} \lambda_{n}^{2}\left|y_{m n}\right|^{2}=\sum_{\lambda_{n} \neq\left|\mu_{m}\right|} \frac{\lambda_{n}^{2}\left|f_{m n}\right|^{2}}{\left(\lambda_{n}^{2}-\left|\mu_{m}\right|^{2}\right)^{2}} \\
& \quad \leq \frac{1}{d^{2}} \sum_{\lambda_{n} \neq\left|\mu_{m}\right|} \lambda_{n}^{2}\left|f_{m n}\right|^{2}=\frac{1}{d^{2}}\left|f_{x}\right|_{L^{2}(Q)}^{2} .
\end{aligned}
$$

Similarly

$$
\left|y_{t}\right|_{L^{2}(Q)}^{2} \leq \frac{1}{d^{2}} \sum_{\lambda_{n} \neq\left|\mu_{m}\right|}\left|\mu_{m}\right|^{2} f_{m n}^{2}=\frac{1}{d^{2}}\left|f_{t}\right|_{L^{2}(Q)}^{2},
$$

so (2.26) is proved, too. 
In order to prove that $y=A^{-1} f \in L^{\infty}(Q)$ one first checks that

$$
\sum_{\substack{n=1 \\ \lambda_{n} \neq\left|\mu_{m}\right|}}^{\infty} \frac{1}{\left.\left|\lambda_{n}^{2}-\right| \mu_{m}\right|^{2} \mid} \leq C,
$$

where $C$ is a constant independent of $m$.

On the other hand, by $(2.31)$

$$
\left.\left.\left|\lambda_{n}^{2}-\right| \mu_{m}\right|^{2}\right|^{2} \geq\left. d\left|\lambda_{n}^{2}-\right| \mu_{m}\right|^{2}\left|, \quad \lambda_{n} \neq\right| \mu_{m} \mid,
$$

so

$$
\sum_{\substack{n=1 \\ \lambda_{n} \neq\left|\mu_{m}\right|}}^{\infty} \frac{1}{\left.\left.\left|\lambda_{n}^{2}-\right| \mu_{m}\right|^{2}\right|^{2}} \leq C / d
$$

It follows that

$$
\sum_{\substack{n=1 \\ \lambda_{n} \neq\left|\mu_{m}\right|}}^{\infty} \frac{\left|f_{m n}\right|}{\left.\left|\lambda_{n}^{2}-\right| \mu_{m}\right|^{2} \mid} \leq C d^{-1} \sum_{n}\left|f_{m n}\right|^{2} .
$$

Therefore the series of $y$ is bounded in $L^{\infty}(Q)$, namely:

$$
|y(x, t)| \leq \sum_{m} \sum_{\substack{n=1 \\ \lambda_{n} \neq\left|\mu_{m}\right|}} \frac{\left|f_{m n}\right|}{\left.\left|\lambda_{n}^{2}-\right| \mu_{m}\right|^{2} \mid} \leq C d^{-1}\left(\sum_{m} \sum_{n}\left|f_{m n}\right|^{2}\right) \leq C d^{-1}|f|_{L^{2}(Q)}^{2} .
$$

This implies (2.25).

Finally, $D(A)$ contains $C_{\pi}^{2}(\bar{Q})$, which is dense in $L^{2}(Q), A$ is symmetric and (2.27) holds. Therefore $A$ is selfadjoint, and the proof is complete.

\section{THE NONLINEAR EQUATION}

We are now in a position to give the main results on (1.1).

Definition 3.1. The function $y \in L^{2}(Q)$ is said to be a weak solution to (1.1) in $Q$ if

$$
\int_{Q} y A_{0} \varphi d x d t+\int_{Q} g(y) \varphi d x d t=\int_{Q} f \varphi d x d t
$$

for all $\varphi \in C_{\pi}^{2}(\bar{Q})$ as indicated in (2.18), with $A_{0}$ defined in (2.15).

The last assumption on $f$ and $g$ is

(H3) $f \in L^{\infty}(Q)$, and

$$
g(-\infty)+\delta \leq u(x)\left(P\left(u^{-1} f\right)\right)(x, t) \leq g(+\infty)-\delta, \quad \text { a.e. }(x, t) \in Q,
$$

for some $\delta>0$. Here $P: L^{2}(Q) \rightarrow N(A)$ is the projection operator on $N(A)$.

We are now in a position to state the main result of this paper, namely

Theorem 3.1. Assume that $T$ is a rational multiple of $\pi$ as in (1.9) and that Hypotheses (H1), (H2) and (H3) are fulfilled with

$$
0<\gamma<\alpha, \quad \alpha=\inf \left\{\left|\mu_{m}\right|^{2}-\lambda_{n}^{2} ; \lambda_{n}<\left|\mu_{m}\right|\right\} .
$$

Then equation (1.1) has at least one weak solution $y \in L^{\infty}(Q)$. This weak solution is unique modulo $N(A)$, i.e., if $y$ and $z$ are weak solutions of (1.1), then $y-z \in N(A)$. If $g$ is strictly increasing, then the weak solution of (1.1) is unique. 
Proof. Denote by $\tilde{G}$ the realization of $g$ in $L^{2}(Q)$, i.e.,

$$
(\tilde{G}(y))(x, t)=g(y(x, t)) \quad \text { a.e. }(x, t) \in Q, y \in L^{2}(Q),
$$

and set $G=u^{-1} \tilde{G}$. In view of (H2), $G: L^{2}(Q) \rightarrow L^{2}(Q)$ is a (continuous and) monotone operator, i.e.,

$$
\langle G(y)-G(z), y-z\rangle \geq 0, \quad \forall y, z \in L^{2}(Q),
$$

where $\langle\cdot, \cdot\rangle$ denotes the inner product in $L^{2}(Q)$ as defined by (1.7). Moreover,

$$
|G(y)-G(z)|^{2} \leq \gamma\langle G(y)-G(z), y-z\rangle, \quad \forall y, z \in L^{2}(Q) .
$$

Replacing $g$ in (1.4) by $g+\varepsilon$ (with $\varepsilon>0$ ) one obtains (on the basis of monotonicity of $y \rightarrow g(y)+\varepsilon y)$

$$
|g(y)+\varepsilon y-(g(z)+\varepsilon z)| \leq(\gamma+\varepsilon)|y-z|, \quad \forall y, z \in \mathbb{R},
$$

and accordingly

$$
\begin{aligned}
& |(G+\varepsilon I)(y)-(G+\varepsilon I)(z)|^{2} \\
& \quad \leq(\gamma+\varepsilon)\langle(G+\varepsilon) y-(G+\varepsilon) z, y-z\rangle
\end{aligned}
$$

for all $y, z \in L^{2}(Q)$.

On the basis of (3.5) and (3.6), the following additional properties of $G$ hold:

$$
\begin{aligned}
& R(G+\varepsilon I)=L^{2}(Q), \\
& \left\langle(G+\varepsilon I)^{-1} y-(G+\varepsilon I)^{-1} z, y-z\right\rangle \geq(\gamma+\varepsilon)^{-1}|y-z|^{2}, \quad \forall y, z \in L^{2}(Q) .
\end{aligned}
$$

In terms of $A$ and $G, y$ is a weak solution to (1.1) in $Q$ (i.e., (3.1) holds) if and only if

$$
A y+G(y)=u^{-1} f .
$$

In order to take advantage of the invertibility of $G+\varepsilon I$ we will consider the following approximation of (3.7), namely

$$
A y+(G+\varepsilon I) y=u^{-1} f, \quad y \in L^{2}(Q),
$$

which can be equivalently written (using an idea of Brézis [4]) as

$$
A^{-1} v+(G+\varepsilon I)^{-1}\left(u^{-1} f+v\right) \in N(A), \quad v \in R(A),
$$

where $A^{-1} \in L(R(A))$ is the operator defined in Proposition 2.1.

Indeed, if $y$ satisfies (3.8) (i.e., (3.1)) and $y_{1} \in N(A), y_{2} \in R(A)$ are the components of $y$ in $N(A) \oplus R(A)=L^{2}(Q)$ (i.e., $\left.y=y_{1}+y_{2}\right)$, then (3.8) yields $(G+\varepsilon I) y=u^{-1} f-A y_{2}$. Set $A y_{2}=v \in R(A)$, i.e., $y_{2}=A^{-1} v$. Then $y=$ $(G+\varepsilon I)^{-1}\left(u^{-1} f-v\right)=y_{1}+A^{-1} v$, which shows that (3.8) and (3.9) are equivalent.

On the other hand, (3.9) is equivalent to

$$
A^{-1} v+(G+\varepsilon I)^{-1}\left(u^{-1} f+v\right)+\partial J(v) \ni 0, \quad v \in R(A),
$$

where $J$ is the indicator function of $R(A)$ (i.e., $J(v)=0$ if $v \in R(A)$ and $J(v)=+\infty$ if $v \notin R(A)$ ), and $\partial J$ is the subdifferential of $J$. Taking into account that $\partial J(v)$ is the cone of the normals to $R(A)$ at $v$, it follows that $\partial J(v)=N(A)$, for all $v \in R(A)$.

Finally, (2.24) shows that $A^{-1}+\alpha^{-1} I$ is monotone on $R(A)$, so (3.10) can be rewritten in the equivalent form

$$
\left(A^{-1}+\alpha^{-1} I\right) v+G_{\alpha}(v)+\partial J(v) \ni 0, \quad v \in R(A),
$$


with $G_{\alpha} v=(G+\varepsilon I)^{-1}\left(u^{-1} f+v\right)-\alpha^{-1} v$. In view of $(3.6)^{\prime}, G_{\alpha}$ satisfies

$$
\left\langle G_{\alpha} v_{1}-G_{\alpha} v_{2}, v_{1}-v_{2}\right\rangle \geq\left((\gamma+\varepsilon)^{-1}-\alpha^{-1}\right)\left|v_{1}-v_{2}\right|^{2}, \quad v_{1}, v_{2} \in R(A) .
$$

We now prove that (3.11) has a solution $v_{\varepsilon}$ for each $\varepsilon<\alpha-\gamma$.

On the basis of (3.12), for $\varepsilon<\alpha-\gamma, G_{\alpha}$ is coercive and maximal monotone in $L^{2}(Q)$ (being continuous and monotone).

A key step now is to prove that the monotone operator $v \rightarrow A_{\alpha} v+\partial J(v)$ with $A_{\alpha}=A^{-1}+\alpha^{-1} I, D\left(A_{\alpha}\right)=R(A)$ and $\partial J=N(A)$ is maximal monotone in $L^{2}(Q)$, i.e., for every $h \in L^{2}(Q)$, the equation (inclusion)

$$
v+A_{\alpha} v+\partial J(v) \ni h
$$

has a solution $v \in R(A)$. Indeed, this equation is equivalent to

$$
v+A_{\alpha} v=(I-P) h, \quad v \in R(A),
$$

which has a unique solution $v \in R(A)$ (as $A_{\alpha}$ is continuous and monotone from $R(A)$ into itself). It follows that $\left(A_{\alpha}+\partial J\right)+G_{\alpha}$ is maximal monotone in $L^{2}(Q)$. Moreover, as $G_{\alpha}$ is coercive, $A_{\alpha}+\partial J+G_{\alpha}$ is onto. Therefore (3.11) has a solution $v_{\varepsilon} \in R(A)$ which is a solution of (3.9). This means that there is $y_{\varepsilon}^{1} \in N(A)$ such that $A^{-1} v_{\varepsilon}+(G+\varepsilon I)^{-1}\left(u^{-1} f+v_{\varepsilon}\right)=y_{\varepsilon}^{1}$. Set $y_{\varepsilon}^{2}=A^{-1} v_{\varepsilon}$. Then $y_{\varepsilon}=y_{\varepsilon}^{1}-y_{\varepsilon}^{2}$ is a solution of (3.8).

We now prove that the solution $y_{\varepsilon}$ of $(3.8)$, i.e.,

$$
\varepsilon y_{\varepsilon}+A y_{\varepsilon}+G y_{\varepsilon}=u^{-1} f
$$

is bounded in $L^{\infty}(Q)$. To this aim, we note that by the assumption (H3)

$$
u(x)\left(P\left(u^{-1} f\right)\right)(x, t) \subset K \subset \operatorname{int} R(g), \quad \text { a.e. }(x, t) \in Q,
$$

where $K$ is a compact interval. Hence, there is $\xi=\xi(x, t)$ with $|\xi| \leq C$, such that

$$
u(x, t)\left(P\left(u^{-1} f\right)\right)(x, t)+\delta w=g(\xi), \quad \text { a.e. }(x, t) \in Q,
$$

for all $\delta>0$ sufficiently small and $|w|=1$. Then, the monotonicity of $g$ yields

$$
\left(g\left(y_{\varepsilon}\right)-u P\left(u^{-1} f\right)-\delta w\right)\left(y_{\varepsilon}-\xi\right) \geq 0, \quad \text { a.e. }(x, t) \in Q,
$$

with $g\left(y_{\varepsilon}(x, t)\right)=u(x, t)\left(G y_{\varepsilon}\right)(x, t)$ (by $\left.(3,2)\right)$, so

$$
\delta w y_{\varepsilon} \leq\left(g\left(y_{\varepsilon}\right)-u P\left(u^{-1} f\right)\right) y_{\varepsilon}-\xi\left(g\left(y_{\varepsilon}\right)-g(\xi)\right), \quad \text { a.e. }(x, t) \in Q
$$

which implies (for $w=y_{\varepsilon}(x, t) /\left|y_{\varepsilon}(x, t)\right|$ ) that

$$
\delta\left|y_{\varepsilon}\right|_{L^{1}(Q)} \leq\left\langle G y_{\varepsilon}-P\left(u^{-1} f\right), y_{\varepsilon}\right\rangle+C\left|G y_{\varepsilon}\right|+C_{1}
$$

for some positive constants $C$ and $C_{1}$.

On the other hand, in view of $L^{2}(Q)=N(A) \oplus R(A)$ (in (2.27)), $u^{-1} f=$ $P\left(u^{-1} f\right)+A y_{1}$ with $y_{1} \in D(A)$ and $u P\left(u^{-1} f\right)=g(z)=u G(z)$ (according to (3.16) and $(3.2))$ for some $z=z(x, t)$ in $L^{\infty}(Q)$. Therefore, (3.15) can be written as

$$
\varepsilon y_{\varepsilon}+A\left(y_{\varepsilon}-y_{1}\right)+G\left(y_{\varepsilon}\right)-G(z)=0
$$

with $G(z)=P\left(u^{-1} f\right)$ and

$$
\begin{aligned}
& \left\langle A\left(y_{\varepsilon}-y_{1}\right), u_{\varepsilon}-y_{1}\right\rangle \geq-\alpha^{-1}\left|A\left(y_{\varepsilon}-y_{1}\right)\right|^{2}, \\
& \left\langle G\left(y_{\varepsilon}\right)-G(z), y_{\varepsilon}-z\right\rangle \geq \gamma^{-1}\left|G y_{\varepsilon}-G z\right|^{2},
\end{aligned}
$$


with $\gamma^{-1}>\alpha^{-1}$. An elementary (but not immediate) combination of (3.20)-(3.22) jointly with an inequality of the form

$$
a b \leq \varepsilon a^{2}+(4 \varepsilon)^{-1} b^{2}, \quad \forall \varepsilon>0, \quad a, b \in R,
$$

leads to the boundedness of $\left|A y_{\varepsilon}\right|$ and $\left|G y_{\varepsilon}\right|$. On the other hand, by (3.20) $\delta\left|y_{\varepsilon}\right|_{L^{1}(Q)}$ can be estimated in terms of $\left|A y_{\varepsilon}\right|$ and $\left|G y_{\varepsilon}\right|$. Indeed,

$$
\begin{aligned}
\left\langle G y_{\varepsilon}-G z, y_{\varepsilon}\right\rangle & =\left\langle-\varepsilon y_{\varepsilon}-A\left(y_{\varepsilon}-y_{1}\right), y_{\varepsilon}\right\rangle \leq-\left\langle A\left(y_{\varepsilon}-y_{1}\right), y_{\varepsilon}\right\rangle \\
& \leq \alpha^{-1}\left|A\left(y_{\varepsilon}-y_{1}\right)\right|^{2}-\left\langle A\left(y_{\varepsilon}-y_{1}\right), y_{1}\right\rangle \leq C .
\end{aligned}
$$

Going back to (3.20), we get

$$
\left|y_{\varepsilon}\right|_{L^{1}(Q)} \leq C, \quad \forall \varepsilon>0 .
$$

It is now easy to prove that $\left|y_{\varepsilon}\right|_{L^{\infty}(Q)}$ is bounded. To this goal, write $y_{\varepsilon}=y_{\varepsilon}^{1}+y_{\varepsilon}^{2}$ with $y_{\varepsilon}^{1} \in N(A)$ and $y_{\varepsilon}^{2} \in R(A)$. Since $A y_{\varepsilon}=A y_{\varepsilon}^{2}$ is bounded in $L^{2}(Q), y_{\varepsilon}^{2}$ is bounded in $L^{\infty}(Q)$ (by (2.25)). Consequently $y_{\varepsilon}^{1}=y_{\varepsilon}-y_{\varepsilon}^{2}$ is bounded in $L^{1}(Q)$, so its Fourier coefficients $y_{\varepsilon m n}^{1}=\int_{Q} y_{\varepsilon}^{1}(x, t) u(x) \varphi_{n}(t) \psi_{m}(t) d x d t$ are bounded as $\left|\varphi_{n}(x)\right| \leq C,\left|\psi_{m}(t)\right| \leq C$ for some $C$ independent of $m, n, x$ and $t$. Therefore $\left|y_{\varepsilon m n}^{1}\right| \leq C\left|y_{\varepsilon}^{1}\right|_{L^{1}(Q)} \leq C_{1}$. Taking into account that $N(A)$ is finite dimensional, it follows that $y_{\varepsilon}^{1}$ is bounded in $L^{\infty}(Q)$, and hence so is $y_{\varepsilon}$. We now show that $\left\{A y_{\varepsilon}\right\}$ and $\left\{G y_{\varepsilon}\right\}$ are Cauchy sequences in $L^{2}(Q)$. To this goal, set $z_{\varepsilon \lambda}=\varepsilon y_{\varepsilon}-\lambda y_{\lambda}$. Clearly, $z_{\varepsilon \lambda} \rightarrow 0$ in $L^{2}(Q)$ as $\lambda, \varepsilon \rightarrow 0$. On the other hand, from (3.15) we have

$$
\left\langle A\left(y_{\varepsilon}-y_{\lambda}\right), y_{\varepsilon}-y_{\lambda}\right\rangle+\left\langle G\left(y_{\varepsilon}\right)-G\left(y_{\lambda}\right), y_{\varepsilon}-y_{\lambda}\right\rangle \leq C\left|z_{\varepsilon \lambda}\right| .
$$

An obvious combination of (3.25), (3.4) and (2.24) leads to

$$
\gamma^{-1}\left|G\left(y_{\varepsilon}\right)-G\left(y_{\lambda}\right)\right|^{2} \leq C\left|z_{\varepsilon \lambda}\right|+\alpha^{-1}\left|A\left(y_{\varepsilon}-y_{\lambda}\right)\right|^{2} .
$$

Finally (3.26) in conjunction with $A\left(y_{\varepsilon}-y_{\lambda}\right)=G\left(y_{\lambda}\right)-G\left(y_{\varepsilon}\right)-z_{\varepsilon \lambda}$ and $\gamma \alpha^{-1}<1$ implies that $\left|G\left(y_{\lambda}\right)-G\left(y_{\varepsilon}\right)\right| \rightarrow 0$ as $\lambda, \varepsilon \rightarrow 0$, and therefore $A\left(y_{\varepsilon}-y_{\lambda}\right)$ is also a Cauchy sequence in $L^{2}(Q)$. The sequence $\left\{y_{\varepsilon}\right\}$ is bounded in $L^{2}(Q)$, so it contains a weakly convergent subsequence (denoted again by $\left\{y_{\varepsilon}\right\}$ for simplicity). Say $y_{\varepsilon} \rightarrow y$ (weakly) in $L^{2}(Q)$. Taking into account that $G$ is maximal monotone in $L^{2}(Q)$ (being continuous and monotone) and that $G y_{\varepsilon}$ is strongly convergent in $L^{2}(Q)$, it follows that $G\left(y_{\varepsilon}\right) \rightarrow G(y)$ (strongly) in $L^{2}(Q)$. Finally, it follows that $y \in D(A)$, $A y_{\varepsilon} \rightarrow A y$, and, letting $\varepsilon \downarrow 0,(3.15)$ implies (3.7).

We now can prove that actually $y_{\varepsilon} \rightarrow y$ strongly in $L^{2}(Q)$. Indeed, $A y_{\varepsilon}^{2}=$ $A y_{\varepsilon} \rightarrow A y$ strongly in $L^{2}(Q)$ so $y_{\varepsilon}^{2}=A^{-1}\left(A y_{\varepsilon}\right)$ is also strongly convergent in $L^{2}(Q)$ (say $y_{\varepsilon}^{2} \rightarrow y^{2}$. Then $y^{2} \in R(A)$ ). As $y_{\varepsilon}^{1}=y_{\varepsilon}-y_{\varepsilon}^{2} \rightarrow y-y^{2}$ and $N(A)$ is finite dimensional, it follows that $y_{\varepsilon}^{1} \rightarrow y-y^{2}=y^{1}$ and $y^{1} \in N(A)$. The conclusion is that $y_{\varepsilon} \rightarrow y$ strongly in $L^{2}(Q)$. On the other hand $y_{\varepsilon}$ is bounded in $L^{\infty}(Q)$, so $y \in L^{\infty}(Q)$. Finally, if $y, z$ are two weak solutions of (3.7), then

$$
G(y)-G(z)=-(A y-A z) .
$$

In view of (3.4) and (2.24), this yields

$$
\gamma^{-1}|G(y)-G(z)|^{2}=\gamma^{-1}|A y-A z|^{2} \leq \alpha^{-1}|A(y-z)|^{2}
$$

with $\gamma^{-1}>\alpha^{-1}$. Therefore $A(y-z)=0$, i.e., $y-z \in N(A)$.

If $g$ is strictly increasing then $G$ is one-to-one, so $G(y)-G(z)=0$ implies $y=z$. The proof is complete. 
Corollary 3.1. Let $T=2 \pi \frac{p}{q}$, where $p$ and $q$ are relatively prime positive integers, and let $(\mathrm{H} 1),(\mathrm{H} 2)$ and $(\mathrm{H} 3)$ be fulfilled. If

$$
\rho_{1}=\frac{2}{\pi} \int_{0}^{\pi} \eta_{u}(x) d x<2 p^{-1}+p^{-2} \quad \text { and } \quad 0<\gamma<2 p^{-1}+p^{-2}-\rho_{1},
$$

then equation (1.1) has a unique weak solution $y \in L^{\infty}(Q)$.

Proof. On the basis of Lemma 2.3, in this case $N(A)$ is the trivial space so, according to Theorem 3.1 the weak solution is unique.

We now prove that

$$
\rho_{1}<2 p^{-1}+p^{-2} \quad \text { implies } \quad \alpha \geq 2 p^{-1}+p^{-2}-\rho_{1} .
$$

Set $\mu=p\left(\sqrt{1+\rho_{1}}-1\right)$. We have $\mu<1$ and (for $\left|\mu_{m}\right|>\lambda_{n}$ )

$$
\mu_{m}^{2}-\lambda_{n}^{2}=\frac{1}{p^{2}}\left(|m| q-n p-p \theta_{n}\right)\left(|m| q+n p+p \theta_{n}\right)
$$

with $|m| q>n p+p \theta_{n}$ and $p \theta_{n} \leq \mu<1$ (see (2.21)). Therefore $|m| q \geq n p+1$, which yields

$$
\begin{aligned}
\mu_{m}^{2}-\lambda_{n}^{2} & \geq \frac{1}{p^{2}}\left(1-p \theta_{n}\right)\left(2 p+1+p \theta_{n}\right) \\
& \geq \frac{1}{p^{2}}(1-\mu)(2 p+1+\mu)=2 p^{-1}+p^{-2}-\rho_{1},
\end{aligned}
$$

and, in turn,

$$
\alpha=\inf \left\{\mu_{m}^{2}-\lambda_{n}^{2},\left|\mu_{m}\right|>\lambda_{n}\right\} \geq 2 p^{-1}+p^{-2}-\rho_{1} .
$$

Therefore, it suffices to choose

$$
0<\gamma<2 p^{-1}+p^{-2}-\rho_{1},
$$

which completes the proof.

Note that $u=1$ (or more generally $\eta_{u}=0$, i.e., $\left.u=\left(c_{1} x+c_{2}\right)^{2}\right)$ implies $\rho_{1}=0$; so for $p=1$, (3.33) contains the well known condition $\gamma<3$ (see [4]).

Remark 3.1. 1) A careful examination of the proof of Theorem 3.1 shows that the results of this section remain valid under the more general assumption that $g=g(x, y)$ is continuous and nondecreasing in $y \in \mathbb{R}, x \rightarrow g(x, y) \in L^{\infty}$, and

$$
|g(x, r)-g(x, \bar{r})| \leq \gamma|r-\bar{r}|, \quad \forall r, \bar{r} \in \mathbb{R}, \text { a.e. } x \in \mathbb{R} .
$$

Assumption (H3) should be modified as follows:

$$
u(x)\left(P u^{-1} f\right)(x, t) \subset K \quad \text { a.e. }(x, t) \in Q,
$$

where $K$ is a compact interval included in $(g(x,-\infty), g(x,+\infty))$ a.e. $x \in(0, \pi)$.

2) Theorem 3.1 with $y \in L^{2}(Q)$ instead of $L^{\infty}(Q)$ and Proposition 2.1 (except for (2.25), i.e., $A^{-1} f \in L^{\infty}(Q)$ ) remain valid if $T$ is an irrational multiple of $\pi$ of the form $T=2 \pi \alpha, \alpha=\sqrt{p_{0} / q_{0}}$, where $p_{0}$ and $q_{0}$ are relatively prime positive integers such that

$$
\frac{p_{0}}{q_{0}} \neq\left(\frac{m}{n}\right)^{2}, \quad m, n \in \mathbb{N}, \quad \text { and } \quad p_{0} \rho_{1}<1 .
$$

Indeed, in this case $N(A)$ is zero, as

$$
\lambda_{n}^{2}-\mu_{m}^{2}=\left(n+\theta_{n}\right)^{2}-m^{2} \frac{q_{0}}{p_{0}}=p_{0}^{-1}\left(p_{0} n^{2}-q_{0} m^{2}+2 p_{0} n \theta_{n}+p_{0} \theta_{n}^{2}\right)
$$


and, by Lemma 2.1

$$
p_{0}\left(2 n \theta_{n}+\theta_{n}^{2}\right) \leq p_{0}\left(\frac{2 n \rho_{1}}{n+\sqrt{n^{2}+\rho_{1}}}+\frac{\rho_{1}^{2}}{\left(n+\sqrt{n^{2}+\rho_{1}}\right)^{2}}\right) \leq p_{0} \rho_{1}<1 .
$$

This implies $\lambda_{n} \neq \mu_{m}$ and $\inf _{m, n}\left|\lambda_{n}^{2}-\mu_{m}^{2}\right|=d \geq 1-p_{0} \rho_{1}>0$.

In this case (2.40) may not remain valid, so $A^{-1} f$ may not be in $L^{\infty}(Q)$.

In connection with the regularity of the weak solutions of (1.1) we have

Corollary 3.2. In addition to the hypotheses of Theorem 3.1 assume that $f_{t} \in$ $L^{2}(Q)$ and either

1) $\gamma<d, d=\inf \left\{\left|\lambda_{n}^{2}-\mu_{m}^{2}\right|, \lambda_{n} \neq\left|\mu_{m}\right|\right\}$, or

2) for each $r>0$ there is $\rho_{r}>0$ such that

$$
\left|g\left(s_{1}\right)-g\left(s_{2}\right)\right| \geq \rho_{r}\left|s_{1}-s_{2}\right|, \quad \forall s_{1}, s_{2} \in[-r, r] .
$$

Then the weak solutions $y$ of (1.1) are in $H^{1}(Q)$. In the case of (3.34) the weak solution of (1.1) is unique.

Proof. 1) Let $y$ be a weak solution of (1.1), i.e., (3.7) holds: $A y+G(y)=\tilde{f}$ with $\tilde{f}=u^{-1} f$. For a sufficiently small $h$ set

$$
h y^{h}(x, t)=y(x, t+h)-y(x, t), \quad(x, t) \in Q \text { with } t \text { fixed in }(0, T) .
$$

But $y=y_{1}+y_{2}$ with $y_{1} \in N(A)$ and $y_{2} \in R(A)$, so $y^{h}=y_{1}^{h}+y_{2}^{h}$ and $y_{1}^{h} \in N(A)$ is bounded in $L^{\infty}(Q)$ with respect to $h$ (as $N(A)$ is finite dimensional). We have

$$
A y^{h}+G^{h} y=\tilde{f}^{h} ; \quad\left(G^{h} y\right)(x, t)=h^{-1}(g(y(x, t+h))-g(y(x, t))),
$$

and $\left|G^{h} y\right| \leq \gamma\left|y^{h}\right|,\left\langle G^{h} y, y^{h}\right\rangle \geq \gamma^{-1}\left|G^{h} y\right|^{2}$, which yields

$$
\left|y_{2}^{h}\right| \leq\left|A^{-1}\right|\left(\left|\tilde{f}^{h}\right|+\gamma\left|y^{h}\right|\right) \leq d^{-1}\left(\left|f_{t}+C+\gamma\right| y_{2}^{h} \mid\right),
$$

where $\left|A^{-1}\right| \leq d^{-1}$ (by (2.23)) and

$$
\left|\tilde{f}^{h}\right|+\gamma\left|y_{1}^{h}\right| \leq C+\left|\tilde{f}_{t}\right| .
$$

By hypotheses, $d^{-1} \gamma<1$, so (3.36) yields the boundedness of $y_{2}^{h}$ in $L^{2}(Q)$, and therefore $y^{h}$ is bounded in $L^{2}(Q)$, which implies that $y_{t} \in L^{2}(Q)$. This and the fact that $y$ is an weak solution (i.e., (3.1) holds) lead to the conclusion that $y_{x}$ exists too (in the distributional sense) and $y_{x} \in L^{2}(Q)$. Here is the proof.

We have

$$
A y=\tilde{f}-G(y)=F, \quad \text { with } \tilde{f}_{t} \in L^{2}(Q), y_{t} \in L^{2}(Q)
$$

and $(G(y))_{t} \in L^{2}(Q)$. Denote by $P$ the (linear bounded) projection operator on $R(A)$ and by $F^{\varepsilon}$ the usual regularization (mollifier) of $F$. Let $y^{\varepsilon}$ be the solution of $A y^{\varepsilon}=P F^{\varepsilon}$. Therefore $A\left(P y^{\varepsilon}\right)=P F^{\varepsilon}, P F^{\varepsilon} \rightarrow P F=F, A\left(P y^{\varepsilon}\right) \rightarrow F=A y=$ $A P y$; so $P y^{\varepsilon} \rightarrow P y$ as $\varepsilon \downarrow 0$.

By the definition of the weak solution $P y^{\varepsilon}$

$$
\int_{Q} u \varphi_{x}\left(P y_{x}^{\varepsilon}\right) d x d t=\int_{Q} u \varphi_{t}\left(P y_{t}^{\varepsilon}\right) d x d t+\int_{Q} \varphi\left(P F^{\varepsilon}\right) d x d t
$$

Replacing here $\varphi=P y^{\varepsilon}$ and taking into account (2.39), i.e., $P y_{t}^{\varepsilon}\left|\leq \frac{1}{d}\right| P F_{t}^{\varepsilon} \mid$ (which is bounded in $L^{2}(Q)$ by the norm of $f_{t}-(G(y))_{t}$ plus a constant), it follows that 
$P y_{x}^{\varepsilon}$ is bounded in $L^{2}(Q)$. This and $P y^{\varepsilon} \rightarrow P y$ imply $P y_{x} \in L^{2}(q)$ in the sense of distributions. As $N(A)$ is finite dimensional and $(I-P) y \in N(A),(I-P) y$ is in $H^{1}(Q)$. Thus $y_{x} \in L^{2}(q)$, so $y \in H^{1}(Q)$.

2) Condition (3.34) and $y \in L^{\infty}(Q)$ (i.e., $|y(x, t)| \leq r$ a.e. $(x, t) \in Q$, for some $r>0)$ give

$$
\left|h^{-1}\right||g(y(x, t+h))-g(y(x, t))| \geq \rho_{r}\left|y^{h}(x, t)\right|, \quad \text { a.e. }(x, t) \in Q,
$$

for all sufficiently small $h$ (here $t$ is arbitrary in $(0, T)$, but fixed). By the definition of $G$ (e.g. (3.2)) and $G^{h}$ (see (3.35)), it follows that

$$
\left|G^{h} y\right| \geq \rho_{r}\left|y^{h}\right|, \quad\left\langle G^{h} y, y^{h}\right\rangle \geq \gamma^{-1} \rho_{r}^{2}\left|y^{h}\right|^{2} .
$$

Multiplying $A y^{h}+G^{h} y=f^{h}$ by $y^{h}$, using $\left\langle A y^{h}, y^{h}\right\rangle \geq-\alpha^{-1}\left|A y^{h}\right|^{2}$, (3.38) and

$$
\left\langle f^{h}, y^{h}\right\rangle \leq \beta\left|y^{h}\right|^{2}+\frac{1}{4 \beta}\left|f^{h}\right|^{2}
$$

with $0<\beta<\gamma^{-1} \rho_{r}^{2}-\alpha^{-1} \rho_{r}^{2}$, one obtains

$$
\left(\gamma^{-1} \rho_{r}^{2}-\beta\right)\left|y^{h}\right|^{2} \leq \alpha^{-1}\left|A y^{h}\right|^{2}+C
$$

and

$$
\gamma^{-1}\left|G^{h} y\right|^{2} \leq \alpha^{-1}\left|A y^{h}\right|^{2}+\beta\left|y^{h}\right|^{2}+C,
$$

which gives (via (3.39))

$$
\left|G^{h} y\right|^{2} \leq d\left|A y^{h}\right|^{2}+C_{1}
$$

with $d=\alpha^{-1} \rho_{r}^{2} /\left(\gamma^{-1} \rho_{r}^{2}-\beta\right.$ ) (so $d<1$ ) and $C, C_{1}$ independent of $h$. Finally, substituting $G^{h} y=\tilde{f}^{h}-A y^{h}$ into (3.39), we see obviously that $\left|A y^{h}\right|$ is bounded in $L^{2}(Q)$ (with respect to all sufficiently small $h$ ). Going back to (3.39), one gets the boundedness of $y^{h}$ in $L^{2}(Q)$. As seen in 1), this implies $y \in H^{1}(Q)$. The uniqueness of the weak solution follows from Theorem 3.1 and (3.34) (which implies that $g$ is strictly increasing). The proof is complete.

The case $\eta_{u}=0$ (see (1.3)) remains an open problem.

\section{REFERENCES}

[1] A. Bahri and H. Brézis, Periodic solution of a nonlinear wave equation, Proc. Roy. Soc. Edinburgh Sect. A 1-D 85 (1980), 313-320. MR 82f:35011

[2] A. Bamberger, G. Chavent and P. Lailly, About the stability of the inverse problem in wave equations; applications to the interpretation of seismic profiles, Appl. Math. Optimiz. 5 (1979), 1-47. MR 80b:86002

[3] V. Barbu and N. H. Pavel, An inverse problem for the one dimensional wave equation, SIAM J. Control and Optimiz. 35-5 (1997), to appear.

[4] H. Brézis, Periodic solutions of nonlinear vibrating strings and duality principles, Bull. AMS 8 (1983), 409-426. MR 84e:35010

[5] H. Brézis and L. Nirenberg, Forced vibrations for a nonlinear wave equation, Comm. Pure Appl. Math. 31 (1978), 1-30. MR 81i:35112

[6] R. C. Brown, D. B. Hinton and S. Schwabik, Applications of a one-dimensional Sobolev inequality to eigenvalue problems, Differential Integral Equations 9 (1996), 481-498. MR 96k:34180

[7] M. Fečkan, Periodic solutions of certain abstract wave equations, Proc. AMS 123 (1995), 456-471. MR 95c:35030 
[8] P. J. McKenna, On solutions of a nonlinear wave equation when the ratio of the period to the length of the intervals is irrational, Proc. AMS 93 (1985), 59-64. MR 86f:35017

[9] P. Rabinowitz, Free vibrations for a semilinear wave equation, Comm. Pure Appl. Math. 31 (1978), 31-68. MR 84i:35109

[10] K. Yosida, Functional analysis, 6th ed., Springer-Verlag, Berlin, 1980. MR 82i:46002

Department of Mathematics, University of Iasi, Iasi, Romania

E-mail address: barbu@uaic.ro

Department of Mathematics, Ohio University, Athens, Ohio 45701

E-mail address: npavel@bing.math.ohiou.edu 